\title{
A Genotyping-by-sequencing Single Nucleotide Polymorphism-based Map and Genetic Analysis of Root Traits in an Interspecific Tomato Population
}

\author{
Limeng Xie, Patricia Klein, and Kevin Crosby \\ Department of Horticultural Sciences, Texas A\&M University, 2133 TAMU, College Station, \\ TX 77843 \\ John Jifon \\ Texas AgriLife Research and Extension Center, 2415 East Business 83, Weslaco, TX 78596
}

\begin{abstract}
Additional Index words. Solanum cheesmaniae, specific root length, linkage map, quantitative trait loci
ABSTRACT. Roots impact plants' capacity to absorb water and nutrients and thus play a vital role in tolerance to drought, salinity, and nutrient stress. In tomato (Solanum lycopersicum) breeding programs, wild tomato species have been commonly used to increase disease resistance and fruit quality and yield. However, tomato has seldom been bred for water/nutrient use efficiency or resilience to abiotic stress. Meanwhile, little knowledge of the genetic control of root traits in tomato is available. In this study, a mapping population consisting of $181 F_{2}$ progenies derived from a cross between an advanced breeding line RvT1 (S. lycopersicum) and a wild species Lche4 (Solanum cheesmaniae) was evaluated for root and shoot traits in the greenhouse. Root phenotypes were studied for the early seedling stage. Heritability estimates show that root traits are moderately or highly heritable. Root mass was highly correlated with root size (length, surface area, and volume). Shoot mass and chlorophyll content (SPAD) were moderately correlated with root mass and size. Genotyping-by-sequencing was applied to discover single nucleotide polymorphism (SNP) markers. Seven hundred and forty-two SNPs were successfully mapped, and a medium-dense linkage map was created that covered 1319.47 centimorgans $(\mathrm{cM})$ with an average distance of $1.78 \mathrm{cM}$ between adjacent markers. Using composite interval mapping, multiple quantitative trait loci (QTL) mapping and nonparametric mapping, 29 QTLs were identified for 12 root and shoot traits on eight chromosomes. Those QTLs of major and minor effect were involved in the differences among the $F_{2}$ population. Two QTL hotspot regions associated with root mass, size, shoot mass and SPAD were identified on chromosomes 1 and 4 , which was consistent with the correlation among traits. Five QTLs for shoot length and eight QTLs for SPAD were accounting for $\mathbf{4 0 . 0 1 \%}$ and $55.53 \%$ of the phenotypic variation. Two QTLs were associated with $18.26 \%$ of the total variation for specific root length. The wild parent Lche4 has been characterized as a potential genetic donor of higher specific root length and might be a good parent to modify the root system of cultivated tomato.
\end{abstract}

The cultivated tomato is one of the most economically important food crops and a notable component of healthy diets. The world production of tomato was estimated at 182 million tonnes in 2017 (Food and Agriculture Organization of the United Nations, 2017). However, the productivity of tomato is frequently limited by abiotic stresses, including drought, salinity, and nutrient deficiency (Foolad, 2007). The root system plays an essential role in crop resilience to combat biotic and abiotic stresses that ultimately affect yield and quality (Manavalan et al., 2015). Identifying the specific root traits that are important in soil resource (water, nutrients) capture is a major goal in crop improvement for resilience to environmental stresses. Even though many tomato breeding programs have used wild tomato species as sources of disease resistance and improved fruit yield/quality, few investigations have addressed root traits as a key breeding objective. This is probably due to the difficulty of phenotyping, complicating environmental effects, and complex genetic control of root traits (Kuijken et al., 2015). Thus, understanding the genetic

Received for publication 3 Jan. 2019. Accepted for publication 19 Aug. 2019. We thank Natalie Patterson for technical assistance in preparing the genotypingby-sequencing template libraries and Stella Kang for help in phenotyping root traits.

K.C. is the corresponding author. E-mail: k-crosby@tamu.edu. background of key root traits is a prerequisite for developing cultivars with efficient root systems and improved resilience to environmental stresses. For example, $S$. cheesmaniae is a closely related wild tomato species originating from the Galapagos islands, living in the drought-prone and rocky environment, with novel traits, such as high sugar content, rich carotenoid compounds, and whitefly (Bemisia tabaci) resistance (Nuez et al., 2004; Rakha et al., 2017). Previous studies of interspecific population of Solanum Lycopersicum $\times S$. cheesmaniae were mainly focused on fruit weight, soluble solids, $\mathrm{pH}$, and seed mass (Goldman et al., 1995; Paterson et al., 1991). Given the potential of being resistant to drought, its root system is interesting to investigate.

Molecular markers are implemented extensively in breeding programs to assist in the selection of alleles that control traits, such as root traits, that are difficult or expensive to phenotype and can identify QTL for traits of interest (Champoux et al., 1995; Coudert et al., 2010). Many studies on maize (Zea mays), wheat (Triticum aestivum), and rice (Oryza sativa) have successfully mapped numerous QTLs for root development and proved their potential to enhance the yield and stress tolerance in a variety of crop breeding programs (Ali et al., 2000; Bai et al., 2013; Burton et al., 2014; Cai et al., 2012; Liu et al., 2013; Ren et al., 2012 ; Ruta et al., 2010; Topp et al., 2013; Uga et al., 2011). An advanced backcross line developed 
by introducing an exotic QTL controlling root growth angle into a shallow-root rice cultivar, maintained a high yield under a drought environment, compared with the recurrent parent (Uga et al., 2013). Most of these studied root traits showed polygenic control, high genetic variability, and moderate to high heritability.

Tomato has a genome size of $\approx 950$ megabases $(\mathrm{Mb})$ consisting of two parts: gene-poor pericentromeric heterochromatin and gene-rich distal chromatin (Tomato Genome Consortium, 2012). A large number of molecular markers, including restriction fragment length polymorphism (RFLP), simple sequence repeat (SSR), and SNP markers have been developed and applied in construction of high-density linkage maps of tomato. This has allowed extensive QTL mapping for many horticulturally important traits (Foolad, 2007; Sim et al., 2012; Tanksley et al., 1992). Yet there are scarce QTL studies of tomato root traits. One study identified certain chromosome segments regulating root length, growth angle, and some root cellular traits using homozygous introgression lines between $S$. lycopersicum and Solanum pennellii (Ron et al., 2013). Two QTLs have been found related to root ammonia uptake in a backcross population from $S$. lycopersicum and Solanum hirsutum (Truco et al., 2000). The availability of the tomato reference genome and genome annotation information enables efficient discovery of genome-wide SNP markers, allowing more accurate QTL localization and subsequent elucidation of potential candidate genes for important traits (Sim et al., 2012; Tomato Genome Consortium, 2012). Genotyping-by-sequencing (GBS) is one of the powerful tools, yielding thousands of SNP markers in a cost-effective way through its simple library preparation and combining the power of multiplexed nextgeneration sequencing platforms with enzyme-based genome reduction (Elshire et al., 2011; Morishige et al., 2013; Spindel et al., 2013).

The goals of this research were to study phenotypes of root and shoot traits in an $\mathrm{F}_{2}$ interspecific population ( $S$. lycopersicum $\times S$. cheesmaniae), construct a linkage map using GBSSNP markers, and identify QTL for root and shoot traits.

\section{Materials and Methods}

Plant materials. An $\mathrm{F}_{2}$ population consisting of 181 seedlings was the cross between the female parent RvT1 and the male parent Lche4 (S. cheesmaniae, accession LA1035, obtained from the Charles M. Rick Tomato Genetic Resources Center, University of California Davis, CA). RvT1 is a TAMU advanced $\mathrm{F}_{6}$ inbreeding line with favorable horticultural traits, such as red, sweet, saladette-type fruit and Begomovirus resistance. The two parents were characterized by distinct root phenotypes in a preliminary greenhouse experiment (Supplemental Table 1). RvT1 has a thick and long root system, whereas Lche4 has a thin and short root system.

Phenotypic experiment. $F_{2}$ seedlings were grown in a complete randomized design in the greenhouse along with their parents (five replications) and a commercial cultivar Hot-Ty (10 replications) as a commercial hybrid check. The average greenhouse temperature was $26^{\circ} \mathrm{C}$ day $/ 23{ }^{\circ} \mathrm{C}$ night. Seeds were sown on 11 Nov. 2015 in a pure natural play sand media (Pavestone Company, Grapevine, TX) and were planted in six nursery trays with each 220-mL planting cell (Growers Solution LLC, Cookeville, TN). Two cotton balls (U.S. Cotton, Gastonia, NC) were placed at the bottom of every cell to prevent the sand from leaking. Approximately $15 \mathrm{~mL}$ of $20 \mathrm{~N}-$ 8.7P-16.6K fertilizer with micronutrients (Peters Professional, The Scotts Company, Marysville, $\mathrm{OH}$ ) mixed in $3.8 \mathrm{~L}$ of water was applied weekly. The SPAD value of the first three fully expanded leaves was measured on 11-week-old seedlings using a chlorophyll meter (SPAD-502 Plus; Konica Minolta Sensing, Tokyo, Japan; Monje and Bugbee, 1992). At the 12th week after planting, the aboveground part of each plant was cut, and the shoot length (SL) and shoot fresh weight (SFW) were measured. At this stage, the plants had six to eight fully expanded leaves, no flowers, and no fruit sets. Each root tissue was acquired by putting the entire ball on a fine sieve and immersing into the water while lightly shaking the sieve. Next, the root tissue was washed with tap water, preserved in zipper-sealed plastic bags, and stored at $4{ }^{\circ} \mathrm{C}$. Every root sample was floated on a transparent plate filled with a layer of water and scanned with a scanner (Epson Perfection V700 Photo, Epson America, Inc., Long Beach, CA) to export a tiff format image. Some larger root samples, which could not be fully separated on the plate, were divided into two parts to avoid overlap (Liu et al., 2008). After scanning, roots were blotted with a paper towel to remove excess moisture, and the root fresh weight was measured. The dry weight of roots was measured after drying for $72 \mathrm{~h}$ at $80^{\circ} \mathrm{C}$. Scanned root images were analyzed in batch using WinRhizo Pro software (Version 2013a; Regent Instruments Inc., Ottawa, Canada). The root length (RL), root surface area (RSA), root volume (RV), and root average diameter (RAD) were acquired directly by the software. Root-to-shoot ratio (RSR), specific root length (SRL), and root tissue density (RTD) were computed. Trait abbreviation and explanations are described in Table 1.

Statistical analysis. Trait means, standard deviations, ranges, and analysis of variance (ANOVA) tests for parents and the check cultivar were performed in JMP Pro (Version 12.1.0, SAS Institute, Cary, NC). Shapiro-Wilk tests for normality of trait data in the $\mathrm{F}_{2}$ population were performed using RStudio (Version 1.0.136, Boston, MA). Possible associations among the measured traits were tested based on linear Pearson correlation and principal component analysis (PCA). Pearson correction coefficients were visualized by "corrplot" R package (Wei and Simko, 2013). The PCA was carried out using the "princomp" function, and PCA biplot was generated by "ggfortify" R package (Tang et al., 2016). The broad sense heritability of traits was calculated as $\mathrm{H}^{2}=\left[\mathrm{V}_{\mathrm{F} 2}-\left(\mathrm{V}_{\mathrm{p} 1}+\mathrm{V}_{\mathrm{p} 2}+\right.\right.$ $\left.\left.\mathrm{V}_{\text {check }}\right) / 3\right] / \mathrm{V}_{\mathrm{F} 2}$, where the variance of the $\mathrm{F}_{2}$ estimated phenotypic variance, and the average variance of the two parents and the check cultivar was to estimate environmental variance.

GenotyPing. Young leaves of 6-week-old seedlings of the parents and the $181 \mathrm{~F}_{2}$ seedings were sampled in liquid nitrogen and then transferred to $-80{ }^{\circ} \mathrm{C}$. Genomic DNA was extracted after the microprep cetyltrimethyl ammonium bromide protocol with minor adjustments (Fulton et al., 1995). Because tomato leaves contain large amounts of polyphenols, the DNA pellet was further purified with a DNA Clean \& Concentrator kit (ZYMO Research, Irvine, CA) and quantified with a fluorometer (Qubit Fluorometer 2.0, Invitrogen, Carlsbad, CA). NgoMIV (New England Biolabs, Ipswich, MA), a methylation-sensitive restriction enzyme, which cuts sequences of GCCGGC, was used to digest 500 nanograms of DNA of each seedling and two parents. The GBS template library was constructed as described in Morishige et al. (2013). Quality 
Table 1. Summary of measured shoot and root traits of RvT1 (Solanum lycopersicum), Lche4 (S. cheesmaniae), Hot-Ty (S. lycopersicum), and the $\mathrm{F}_{2}$ population of RvT1 $\times$ Lche 4 .

\begin{tabular}{|c|c|c|}
\hline Trait & Trait abbreviation & Trait explained \\
\hline Shoot length (cm) & SL & Distance from shoot tip to shoot bottom \\
\hline Shoot fresh weight $(\mathrm{g})$ & SFW & Shoot fresh weight \\
\hline Chlorophyll content & SPAD & Average chlorophyll content of three first fully expanded leaves \\
\hline Root dry weight (g) & RDW & Root dry weight \\
\hline Root-to-shoot ratio & RSR & Root fresh weight/shoot fresh weight \\
\hline Root length $(\mathrm{cm})$ & RL & Total root length \\
\hline Root avg diameter $(\mathrm{mm})$ & RAD & Average of root diameter \\
\hline Root volume $\left(\mathrm{cm}^{3}\right)$ & RV & Total volume of root tissue \\
\hline Specific root length $\left(\mathrm{m} \cdot \mathrm{g}^{-1}\right)$ & SRL & Ratio of total root length to root dry weight \\
\hline Root tissue density $\left(\mathrm{g} \cdot \mathrm{cm}^{-3}\right)$ & RTD & Ratio of root dry weight to total root volume \\
\hline
\end{tabular}

assessment was performed using a chip-based capillary electrophoresis machine (Agilent 2100 Bioanalyzer; Agilent Technologies, Santa Clara, CA). The template was sequenced on a high-throughput sequencing system (Illumina HiSEq. 2500; Illumina, San Diego, CA) using standard Illumina protocols. Single-end sequencing was carried out for 144 cycles. Only Illumina data that passed quality control was further analyzed. Reads for each parent and seedling were identified by their unique 12 base-pairs (bp) barcode identifier and sorted into individual files using a custom Python script. Perfect matches to both the 12-bp barcode sequence and the partial NgoMIV restriction site were crucial to retain the reads from each sample. Following the sorting of reads to each individual seedling, the 12-bp barcode on the $5^{\prime}$ end was trimmed as were $2 \mathrm{bp}$ from the $3^{\prime}$, resulting in a final read length of $130 \mathrm{bp}$. Trimmed reads from the parental lines were aligned to the $S$. lycopersicum genome assembly release 2.5 downloaded from the Sol Genomics Network. Using BLASTN search tool at National Center for Biotechnology Information (Bethesda, MD) database, polymorphisms between parents were identified and further scored in the seedlings using custom Perl scripts. An Excel file containing the SNP markers with less than $15 \%$ of missing data in the $\mathrm{F}_{2}$ population was generated and used for genetic linkage map construction. The markers were named based on the chromosome they mapped to and the physical position of the SNP.

Genetic MAP CONSTRUCTION. Initially, SNP marker data were imported into JoinMap 4.0 software (Van Ooijen, 2006). The independent logarithm of odds (LOD) was set to 10 for assignment of markers to the 12 linkage groups. The marker order in each linkage group was first fixed according to the physical position of the marker based on alignment to the tomato genome and then determined by the regression mapping algorithm with the following parameters: LOD score $>6$, recombinant frequency $<0.4$, goodness of fit jump threshold equals 5 , and ripple value equals 1 . The Kosambi function was chosen to convert the recombination frequency into the genetic distance, with the assumption that there are some crossover events on the chromosome (Kosambi, 1943). During the subsequent revision process, we used the R/qtl package (Broman et al., 2003) and ASMap (Taylor and Butler, 2014) to assist in identifying problematic markers and individuals. A customized checklist for filtering markers was established. First, all duplicative markers were removed, as well as individuals that shared more than $90 \%$ similarity to another individual or had more than $15 \%$ missing data were checked. Next, the Bonferroni corrected probability value was calculated for the $\chi^{2}$ test of segregation distortion pattern. If there were many markers on a chromosome showing a reduced frequency of one allele, this might indicate real segregation distortion. We chose to keep these markers because multipoint estimations of genetic map distances are little affected by real segregation distortion. For distorted markers surrounded by markers showing normal segregation patterns, we omitted those that were highly skewed $\left(-\log _{10} P\right.$ value $\left.>10\right)$, and the skewed ones $\left(-\log _{10} P\right.$ value $\left.>4.3\right)$ were considered as suspicious markers. Finally, for those markers separated by a physical distance less than 500 bp, one was removed, on the basis of three criteria: percent missing across the population, segregation distortion rate, and number of tight double crossover events. If it was hard to decide which marker to omit, the one resulting in the largest increase in map distance was removed. After using these filtering criteria, the final marker data set was used to create the genetic map based on the initial mapping parameter settings. Suspicious markers were scrutinized one by one if they had deleterious effects on the linkage map. Finally, a heat map of recombination frequency and LOD score was generated to check the general quality of the genetic map.

QTL ANALYSIS. We used three mapping methods: composite interval mapping (CIM), multiple QTL mapping (MQM), and nonparametric interval (NP) mapping (Kruskal-Wallis test statistic), for QTL detection. In CIM, the phenotypic and genotypic data file was loaded into WinQTLCart V2.5 (Wang et al., 2011) running on a standard model with four parameters, set as follows: window size $=10$, the backward regression, walking speed $=1$, and control markers $=5$. MQM was performed in the R/qtl package, following the steps described by Broman and Sen (2009) and Spindel et al. (2013). Initially, we needed to specify a genotyping error rate based on a maximum likelihood estimation method. The preliminary QTL were identified by using the scanone() with the model of Haley-Knott regression. Conditioned on the current QTL model, addqtl() and refineqtl() functions were used to scan for the additional linked QTL and refine the locations of QTL, respectively. Thereafter, the stepwiseqtl() function was used to end the best-fit QTL model by forward selection and backward elimination. The fitqtl() function was run to perform an analysis of variance on the final model for estimating QTL effect and 
Table 2. Statistical summary of root and shoot traits in the $\mathrm{F}_{2}$ population $(\mathrm{n}=181)$ derived from Solanum lycopersicum RvT1 $\times$ S. cheesmaniae Lche4, along with RvT1 $(\mathrm{n}=5)$, Lche4 $(\mathrm{n}=5)$, and Hot-Ty [S. lycopersicum $(\mathrm{n}=10)]$.

\begin{tabular}{|c|c|c|c|c|c|c|c|}
\hline \multirow[b]{2}{*}{ Traits $^{z}$} & \multicolumn{2}{|c|}{ Parents } & \multirow{2}{*}{$\frac{\text { Check }}{\text { Hot-Ty }}$} & \multicolumn{4}{|c|}{$\mathrm{F}_{2}$ Population $^{\mathrm{y}}$} \\
\hline & RvT1 & Lche4 & & Mean \pm SD & Min & Max & $\mathrm{H}^{2}$ \\
\hline $\mathrm{SL}(\mathrm{cm})$ & $17.08 b^{x}$ & $14.96 \mathrm{~b}$ & $23.31 \mathrm{a}$ & $17.64 \pm 4.90$ & 6.60 & 30.20 & $84.20 \%$ \\
\hline SFW (g) & $12.72 \mathrm{~b}$ & $6.02 \mathrm{c}$ & $16.31 \mathrm{a}$ & $9.85 \pm 3.45$ & 1.20 & 17.02 & $61.15 \%$ \\
\hline SPAD & $39.30 \mathrm{~b}$ & $18.74 \mathrm{c}$ & $44.96 \mathrm{a}$ & $29.67 \pm 4.52$ & 17.00 & 44.10 & $77.97 \%$ \\
\hline RFW (g) & $3.75 \mathrm{~b}$ & $0.47 \mathrm{c}$ & $5.06 \mathrm{a}$ & $2.56 \pm 1.57$ & 0.22 & 6.64 & $80.84 \%$ \\
\hline RDW (g) & $0.28 \mathrm{~b}$ & $0.04 \mathrm{c}$ & $0.43 \mathrm{a}$ & 0.1 & 0.02 & 0.48 & $74.59 \%$ \\
\hline RSR & $0.30 \mathrm{a}$ & $0.08 \mathrm{~b}$ & $0.32 \mathrm{a}$ & $0.25 \pm 0.13$ & 0.03 & 0.76 & $80.69 \%$ \\
\hline $\mathrm{RL}(\mathrm{cm})$ & $1467.60 \mathrm{a}$ & $467.01 \mathrm{~b}$ & $1881.14 \mathrm{a}$ & $1596.68 \pm 941.09$ & 143.49 & 535.17 & $87.40 \%$ \\
\hline RSA ( & $246.57 \mathrm{~b}$ & $56.70 \mathrm{c}$ & $329.77 \mathrm{a}$ & $229.84 \pm 131.44$ & 18.29 & 641.11 & $86.03 \%$ \\
\hline $\mathrm{RV}\left(\mathrm{cm}^{3}\right)$ & $3.31 \mathrm{~b}$ & $0.56 \mathrm{c}$ & $4.65 \mathrm{a}$ & $2.67 \pm 1.57$ & 0.15 & 8.21 & $80.04 \%$ \\
\hline RAD (mm) & $0.53 \mathrm{a}$ & $0.39 \mathrm{~b}$ & $0.57 \mathrm{a}$ & $0.46 \pm 0.05$ & 0.32 & 0.61 & $18.73 \%$ \\
\hline $\operatorname{SRL}\left(\mathrm{m} \cdot \mathrm{g}^{-1}\right)$ & $52.92 \mathrm{~b}$ & $107.20 \mathrm{a}$ & $43.08 \mathrm{~b}$ & $89.72 \pm 27.01$ & 35.08 & 175.13 & $57.04 \%$ \\
\hline $\operatorname{RTD}\left(\mathrm{g} \cdot \mathrm{cm}^{-3}\right)$ & $0.086 \mathrm{a}$ & $0.083 \mathrm{a}$ & $0.095 \mathrm{a}$ & $0.074 \pm 0.021$ & 0.046 & 0.168 & $63.63 \%$ \\
\hline
\end{tabular}

${ }^{\mathrm{z}} \mathrm{SL}=$ shoot length, SFW $=$ shoot fresh weight, SPAD = chlorophyll content, RFW = root fresh weight, $\mathrm{RDW}=$ root dry weight, $\mathrm{RSR}=$ root to shoot ratio, $\mathrm{RL}=$ root length, $\mathrm{RSA}=$ root surface area, $\mathrm{RV}=$ root volume, $\mathrm{RAD}=$ root average diameter, $\mathrm{SRL}=$ specific root length, $\mathrm{RTD}=$ root tissue density.

${ }^{\mathrm{y}}$ Min $=$ trait minimum value, $\mathrm{Max}=$ trait maximum value in $\mathrm{F}_{2}$ population, $\mathrm{H}^{2}=$ broad sense heritability.

${ }^{\mathrm{x}}$ All trait means were separated by least significant difference Student's $t$ test at $\alpha=0.05$ level. The same letter indicates there is no significant difference between the two values. proportion of the phenotypic variance explained by each QTL. CIM and MQM are parametric mapping methods, with the assumptions of normally distributed phenotypes. Even though these methods can give reasonable QTL results for skewed phenotypes, nonparametric mapping can also be conducted for crossvalidating and improved efficiency of identifying QTL (Broman and Sen, 2009). Therefore, nonparametric interval mapping was also carried out using the R/qtl software, using the scanone() function with the model of "np." The threshold LOD score for all QTL declarations was determined with 1000 permutations at a $95 \%$ confidence interval, which controls the number of false positives, known as Type I error (Churchill and Doerge, 1994). For each QTL, the nearest flanking markers of a 1-LOD support interval defined by flanking markers are reported.
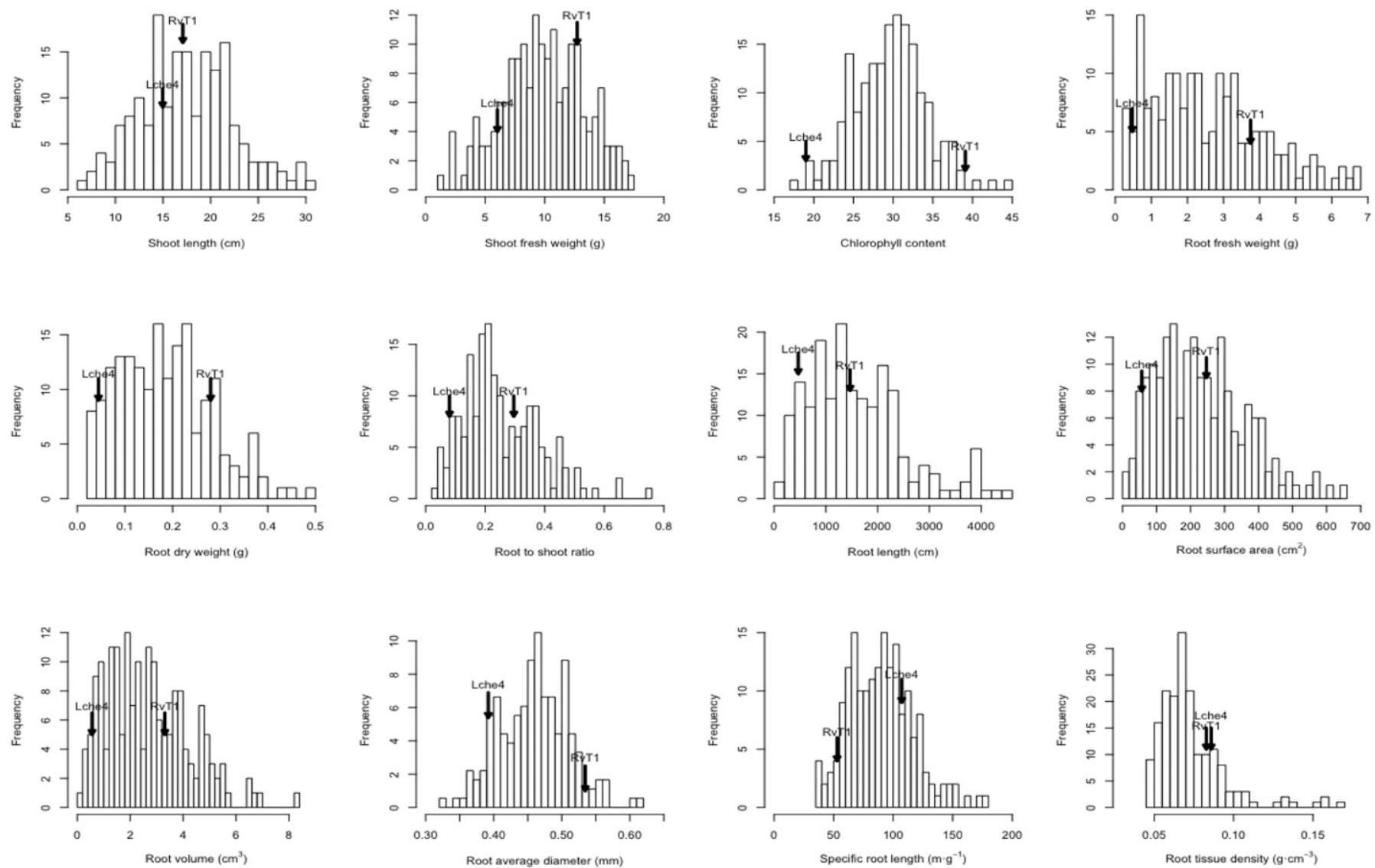

Fig. 1. Frequency distribution of shoot and root traits [shoot length (SL), shoot fresh weight (SFW), chlorophyll content (SPAD), root fresh weigh (RFW), root dry weight (RDW), root-to-shoot ratio (RSR), root length (RL), root surface area (RSA), root volume (RV), root average diameter (RAD), specific root length (SRL) and root tissue density $(\mathrm{RTD})]$ in the $\mathrm{F}_{2}$ population $(\mathrm{n}=181)$ derived from Solanum lycopersicum $\mathrm{RvT} 1 \times$ S. cheesmaniae Lche 4 . Arrows indicate the mean value of RvT1 and Lche4. 


\section{Results}

Phenotypic analysis. The phenotypic values of $181 \mathrm{~F}_{2}$ seedlings, parents and check cultivar are presented in Table 2 . The parents showed significant differences for all measured traits except SL and RTD. The female parent RvT1 had higher values for RFW, RDW, RL, RSA, RV, RAD, and RSR compared with the male parent Lche4. However, the SRL of Lche4 was approximately two times higher than RvT1. Relatively high broad-sense heritability estimates were observed for some traits, including SL (84.20\%), SFW (61.15\%), RFW (80.84\%), RDW (74.59\%), RSR $(80.69 \%)$, RL (87.40\%), RSA (86.03\%), and RV (80.04\%). The population was highly variable and showed a transgressive segregation pattern for all the traits with some phenotypic extremes beyond the parents (Fig. 1). The frequency distributions of shoot traits and RAD were globally normal, which was confirmed by the Shapiro-Wilk test (Supplemental Table 2). The frequency distributions of other root traits were skewed to a lower value.

The measured root traits (RDW, RFW, RV, RSA, RL, and RSR) were significantly positively correlated with each other $(\mathrm{r}>0.7)$ and were grouped together after hierarchical clustering of phenotype based on correlation coefficients in the matrix, suggesting these traits might be controlled by similar genetic factors (Fig. 2). SRL was found to be moderately negatively correlated with RAD $(r=-0.64)$ and RTD $(r=-0.61)$, and slightly negatively correlated with SL and SPAD. There was no significant linear correlation between RTD and any of the shoot traits. SL, SFW, and SPAD showed moderate positive correlations with RDW, RFW, RV, RSA, RL, and RSR. The clustering of trait vectors suggested correlation relationships in the PCA biplot (Fig. 3), which agreed with the preceding correlation analysis. The first two principal components accounted for $53.75 \%$ and $20.12 \%$, collectively accounting for $73.87 \%$ (Fig. 4). The biplot summarized how the traits contributed in a correlated way to characterize different genotypes. The first component relatively associated with shoot traits and RL, RSA, $\mathrm{RV}, \mathrm{RFW}, \mathrm{RDW}$, separating genotypes with small and large biomass, whereas the second component was strongly associated with SRL and RTD, dividing roots with high efficiency or low efficiency.

SNP DISCOVERY AND LINKAGE MAP CONSTRUCTION. After excluding poor-quality markers and markers with more than $15 \%$ missing data, 1002 polymorphic SNP markers from $181 \mathrm{~F}_{2}$ seedlings were obtained. Most SNP markers were located on the upper and lower arms of the chromosomes with less in the gene-poor centromere region, as expected (Supplemental Fig. 1). A spike of marker segregation distortion was found on chromosomes 4 and 11, with a reduced frequency of B alleles from the wild parent Lche4 (Fig. 4). After carefully selecting and scrutinizing markers and individuals, according to our filtering criteria outlined earlier, 742 SNP markers of 181

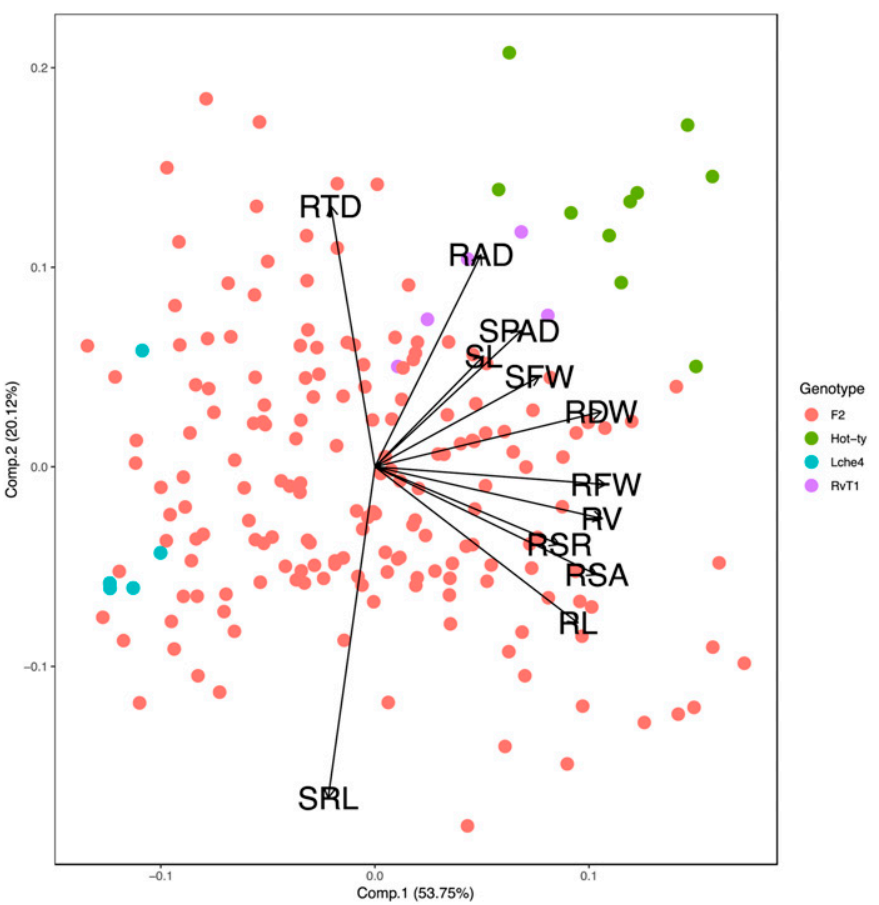

Fig. 3. Biplot generated from principal component analysis of shoot and root traits [shoot length (SL), shoot fresh weight (SFW), chlorophyll content (SPAD), root fresh weigh (RFW), root dry weight (RDW), root-to-shoot ratio (RSR), root length (RL), root surface area (RSA), root volume (RV), root average diameter (RAD), specific root length (SRL), and root tissue density (RTD)] for RvT1 [Solanum lycopersicum $(\mathrm{n}=5)$, purple dot], Lche4 [S. cheesmaniae $(\mathrm{n}=5)$, blue dot], Hot-Ty [S. lycopersicum $(\mathrm{n}=10)$, green dot], and the $\mathrm{F}_{2}$ population of RvT1 $\times$ Lche4 [ $(\mathrm{n}=181)$, red dot]. The first principal component (Comp.1) and the second principal component (Comp.2) explains $53.75 \%$ and $20.12 \%$ of the total variation, respectively. Directional vectors represent traits and points are genotype value. 

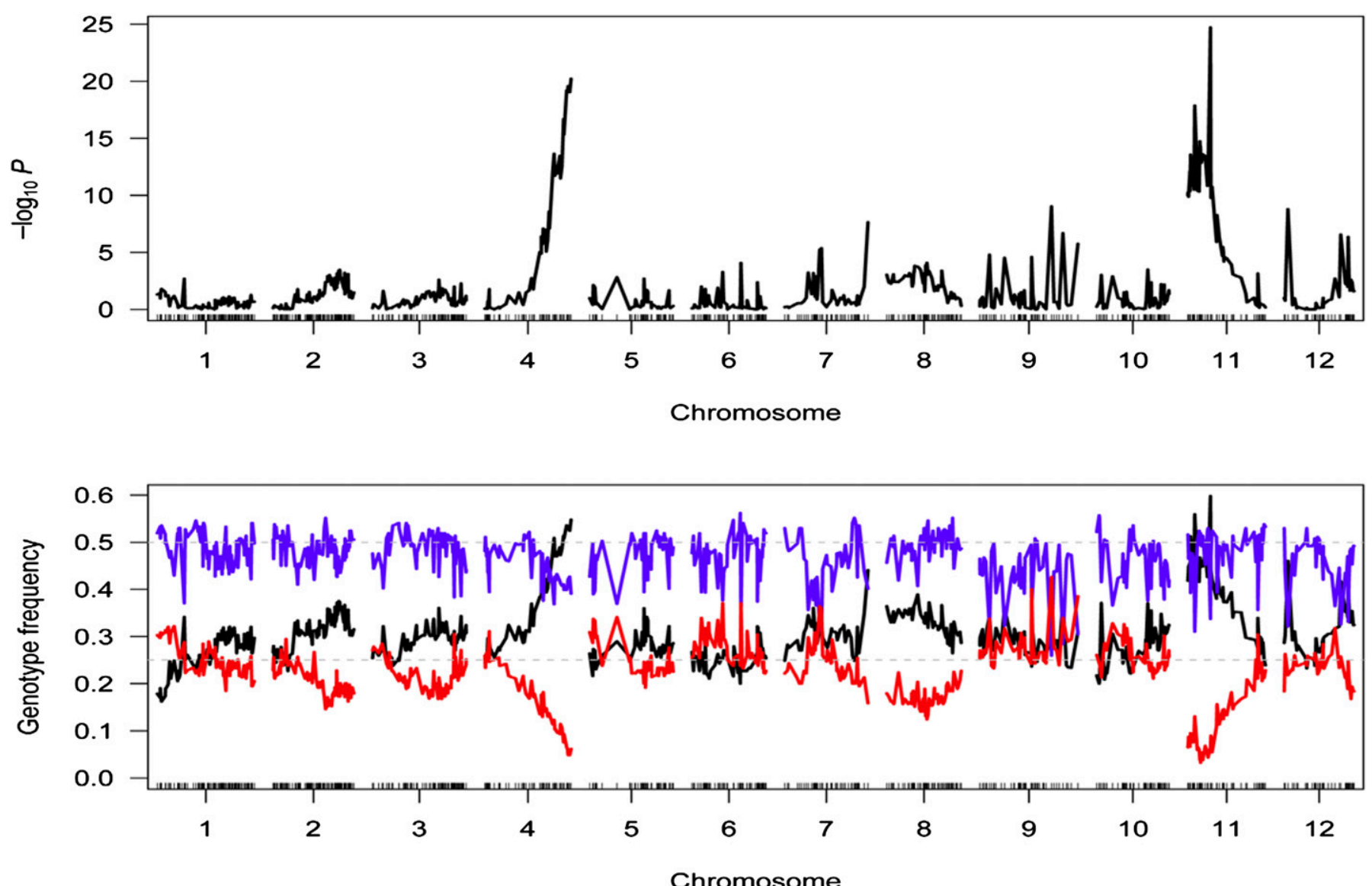

Fig. 4. $-\log _{10} P$ from tests of 1:2:1 segregation ratio at each marker (top panel) and genotype frequencies of each marker. The red, black, and purple lines in the bottom panel denote Lche4 (Solanum cheesmaniae) homozygous alleles, RvT1 (S. lycopersicum) homozygous alleles, and their heterozygous alleles, respectively.

individuals were successfully mapped onto 12 chromosomes, which spanned $1319.47 \mathrm{cM}$. The average distance between two adjacent markers was $1.78 \mathrm{cM}$, and the largest gap between markers was $19.68 \mathrm{cM}$ on chromosome 5 (Table 3, Supplemental Fig. 2). Given the tomato genome size of $\approx 950 \mathrm{Mb}$, the current genetic map is $\approx 0.72 \mathrm{Mb} / \mathrm{cM}$. The heatmap of estimated recombination and LOD scores of mapped markers showed consistent heat across the diagonal line within chromosomes, suggesting this is a good-quality linkage map (Fig. 5).

QTL ANALYSIS. The QTL analysis included $181 \mathrm{~F}_{2}$ seedlings. When two or more QTLs overlapped within the same confidence interval, they were identified as the same QTL. The threshold LOD score declaring a significant QTL was calculated as 3.7 to 4.0 for all traits. A total of 29 putative QTLs for root and shoot traits were detected on nine chromosomes $(1,2$, 3, 4, 6, 8, 9, and 11) by CIM, MQM, and NP (Fig. 6, Table 4). Among them, there were 23 QTLs with higher values that came from the female parent RvT1 and six QTLs with higher values that came from the male parent Lche4. The QTL were named according to the trait name and chromosome location. For example, qSL-6.1 is the first QTL for shoot length on chromosome 6 (Weng et al., 2015). A QTL was defined as a major QTL $\left(R^{2}>10 \%\right)$ or a minor QTL $\left(R^{2}<10 \%\right)$ based on the explained proportion of the phenotypic variation (Weng et al., 2015).
Table 3. Characteristics of the genetic map developed for the Solanum lycopersicum RvT1 $\times S$. cheesmaniae Lche4 $\mathrm{F}_{2}$ population $(\mathrm{n}=181)$.

\begin{tabular}{lcccc}
\hline Chromosome & $\begin{array}{c}\text { Markers } \\
\text { (no.) }\end{array}$ & $\begin{array}{c}\text { Length } \\
(\mathrm{cM})\end{array}$ & $\begin{array}{c}\text { Avg marker } \\
\text { interval }(\mathrm{cM})\end{array}$ & $\begin{array}{c}\text { Maximum } \\
\text { interval }(\mathrm{cM})\end{array}$ \\
\hline Chr1 & 99 & 129.41 & 1.31 & 7.94 \\
Chr2 & 95 & 107.47 & 1.13 & 5.91 \\
Chr3 & 80 & 124.57 & 1.56 & 8.04 \\
Chr4 & 56 & 114.68 & 2.05 & 9.11 \\
Chr5 & 45 & 111.24 & 2.47 & 19.68 \\
Chr6 & 56 & 98.56 & 1.76 & 7.82 \\
Chr7 & 48 & 110.86 & 2.31 & 11.41 \\
Chr8 & 56 & 99.22 & 1.77 & 6.17 \\
Chr9 & 54 & 130.56 & 2.42 & 9.09 \\
Chr10 & 54 & 96.39 & 1.79 & 7.73 \\
Chr11 & 56 & 103.37 & 1.85 & 9.69 \\
Chr12 & 43 & 93.14 & 2.17 & 9.90 \\
Avg & 62 & 109.96 & 1.78 & - \\
Total & 742 & 1319.47 & & - \\
\hline
\end{tabular}

Five QTLs were identified on chromosomes 1, 6, and 11 for SL, together accounting for $40.01 \%$ of the phenotypic variation. Both parents contributed favorably to SL, with RvT1 contributing two minor QTLs and Lche4 contributing three major QTLs. Only one minor QTL on chromosome 4 was found for 


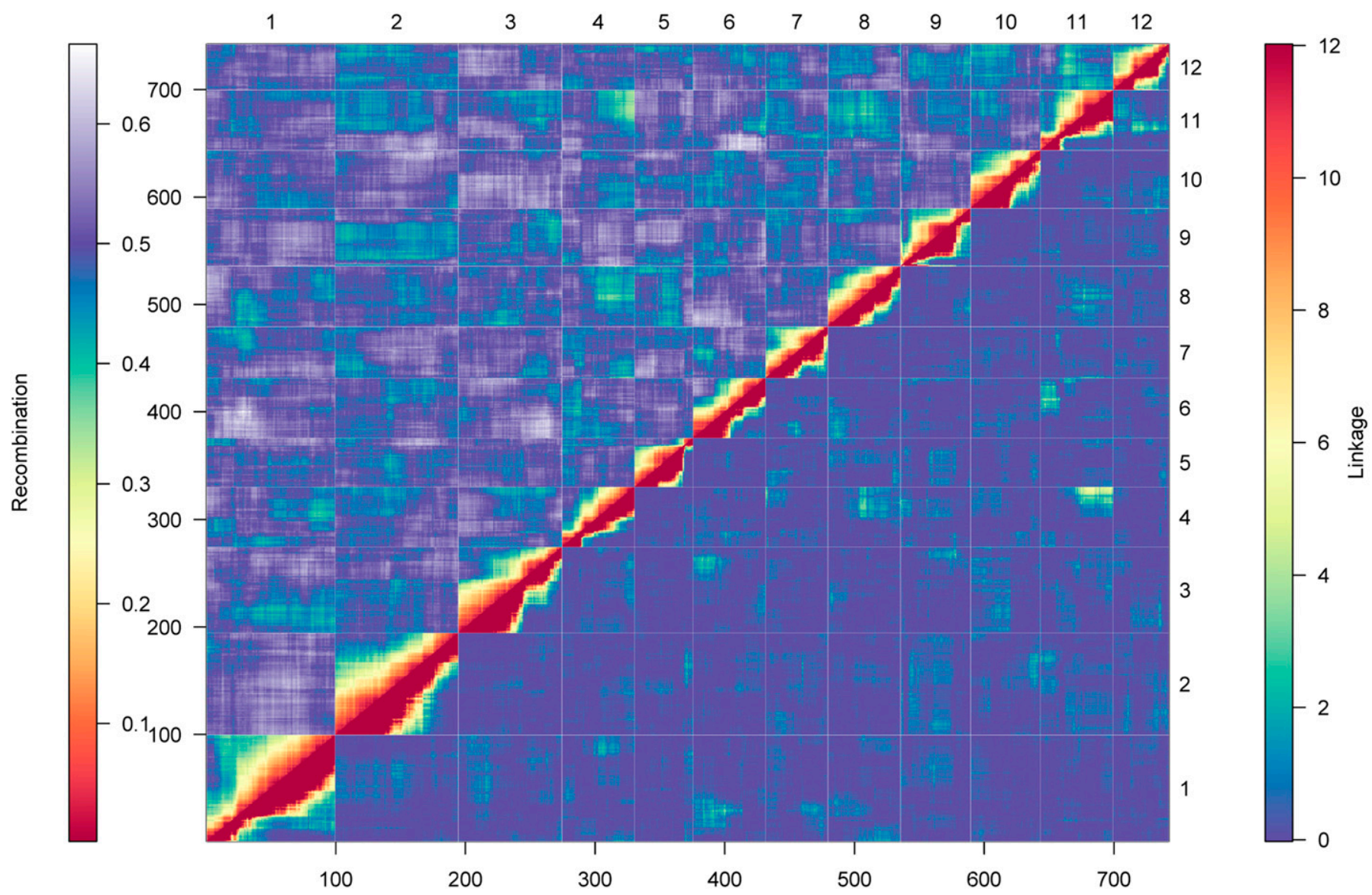

Fig. 5. Heatmap of estimated recombination fractions (upper-left triangle) and logarithm of the odds (LOD) scores (lower-right triangle) for all pairs of markers in the $\mathrm{F}_{2}$ population $(\mathrm{n}=181)$ of Solanum lycopersicum RvT1 $\times$ S. cheesmaniae Lche4. Red indicates markers are linked (large LOD score or small recombination fraction), and blue indicates markers are not linked (small LOD score or large recombination fraction).

SFW, explaining $8.01 \%$ of the variance. For SPAD, eight minor QTLs were observed on chromosomes 1,2, and 8, explaining a combined total of $55.53 \%$ of the phenotypic variance, with alleles from RvT1.

The QTLs for root traits were mainly located on chromosomes 1 and 4. QTLs (qRFW-1, qRFW-4) were detected for RFW on chromosomes 1 and 4 , which explained $11.86 \%$ and $7.73 \%$ of the phenotypic variance, respectively. Similarly, two QTLs (qRDW-1, qRDW-4) were associated with RDW, which explained $9.43 \%$ and $7.80 \%$ of the phenotypic variance, respectively. For RSR, three QTLs were identified on chromosome 1, accounting for a combined total of $69.38 \%$ of the phenotypic variance, with positive alleles contributed by RvT1. One minor QTL for RL on chromosome 4 was detected by nonparametric mapping. Three QTLs for RSA were identified and when combined explained $29.29 \%$ of the phenotypic variance. One major QTL for RV was identified on chromosome 4 explaining $11.12 \%$ of the phenotypic variance. Two QTLs on chromosomes 1 and 8 showing significant associations with SRL totally explained $18.26 \%$ of the phenotypic variance. All alleles increasing SRL were contributed by the male parent Lche4.

Identified QTLs were unevenly distributed on chromosomes, with two major QTL clusters on chromosomes 1 and chromosome 4 (Fig. 6, Table 4). Eight QTL (qSL-1, qSPAD1.1, qSPAD-1.2 qRFW-1, qRDW-1, qRSR-1.1, qRSR-1.2, and
qRSR-1.3) were located on chromosome 1 between 5.6 and $40.8 \mathrm{cM}$. The second major QTL cluster containing five QTL for RFW, RDW, RL, RSA, and RV was located on chromosome 4 between 28.1 and $40.8 \mathrm{cM}$. These five QTLs were mainly dominant (Table 4).

\section{Discussion}

Trait variation. The present study has shown that the $F_{2}$ population derived from a cross of the cultivated tomato and the wild species $S$. cheesmaniae has genetic variability for root morphological traits. Although the wild parent Lche4 had smaller root and shoot biomass than RvT1, it had a much higher SRL, which might be because Lche4 originated in an arid habitat. Many species that live in unproductive, nutrientlimited, or dry environments have been observed to have increased SRL, compared with the species in productive environments (Ryser, 2006). One explanation for higher SRL in plants is that with a relatively small initial investment in biomass per unit RL, the plants could explore larger soil volumes and higher resource uptake rate with higher efficiency (Eissenstat, 1991; Hajek et al., 2013). A higher SRL could be achieved either by reducing RTD or decreasing RAD in plants (Hajek et al., 2013), which was also consistent with our trait correlation analysis. It is important to identify positive and negative correlations among traits because selection for one 


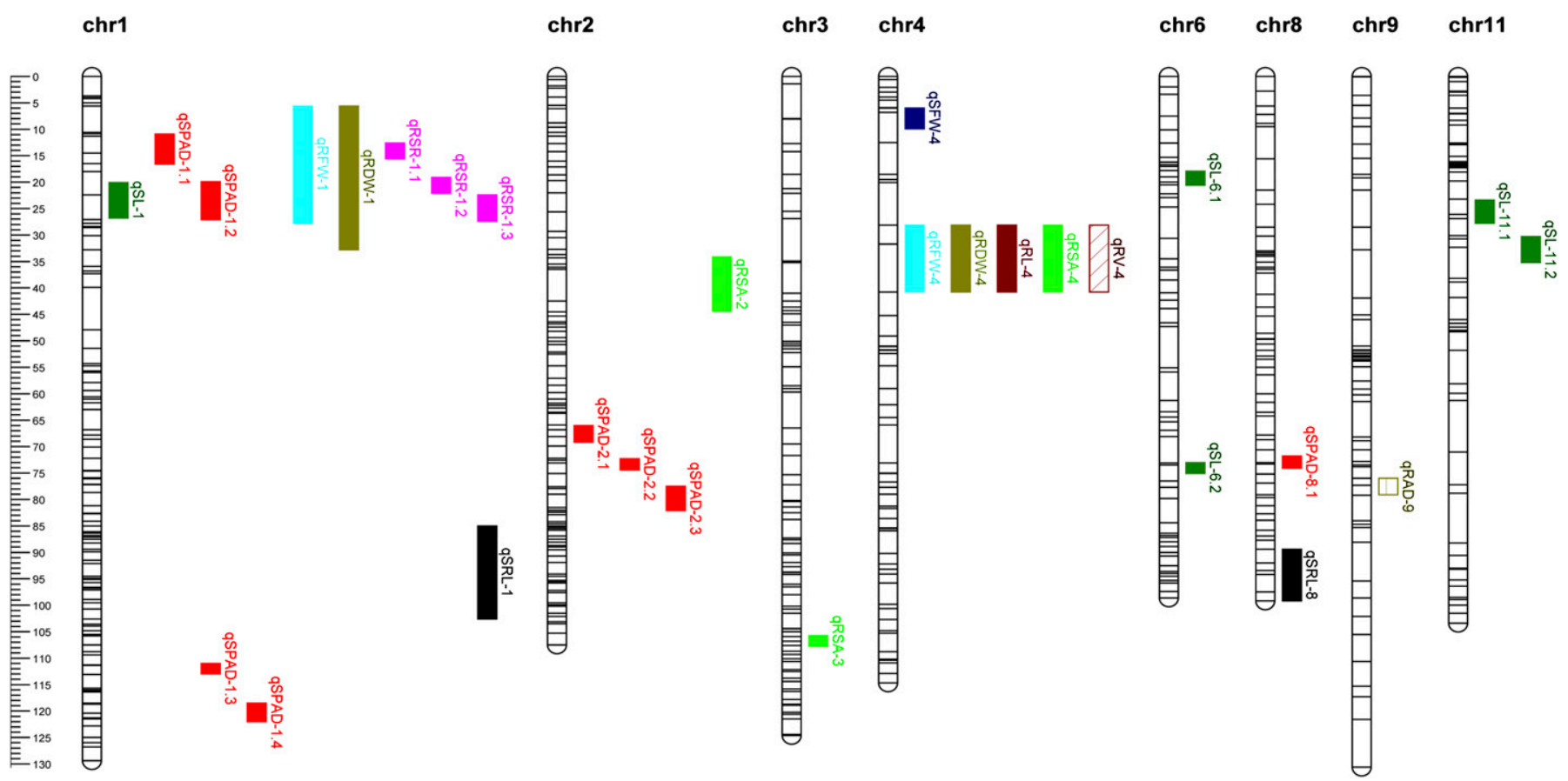

Fig. 6. Quantitative trait loci (QTL) for root and shoot traits [shoot length (SL), shoot fresh weight (SFW), chlorophyll content (SPAD), root fresh weigh (RFW), root dry weight (RDW), root-to-shoot ratio (RSR), root length (RL), root surface area (RSA), root volume (RV), root average diameter (RAD), specific root length $(\mathrm{SRL})$, and root tissue density (RTD)] located on eight chromosomes (Chr) of Solanum lycopersicum $\mathrm{RvT} 1 \times$ S. cheesmaniae Lche4 $\mathrm{F}_{2}$ genetic map. QTL are named and represented by 1-logarithm of the odds (1-LOD) interval. QTL are color-coded according to trait: $\mathrm{SL}=$ dark green, $\mathrm{SFW}=$ dark blue, $\mathrm{SPAD}=$ red, $\mathrm{RFW}=$ cyan, $\mathrm{RDW}=$ army green, $\mathrm{RSR}=$ magenta, $\mathrm{RL}=$ maroon, $\mathrm{RSA}=$ green, $\mathrm{RV}=$ maroon dot, $\mathrm{RAD}=$ army green solid line, $\mathrm{SRL}=$ black.

trait may affect selection for other desirable traits. The moderately positive correlation between shoot mass traits (SL and SFW) with root mass traits (RFW, RDW, RL, RSA, and $\mathrm{RV}$ ) indicates that increasing root mass will not decrease shoot mass, whereas selecting higher SRL could slightly reduce chlorophyll content $(\mathrm{r}=-0.19)$. Significant variations and a continuous distribution for the measured root and shoot traits suggested that this population was suitable for our QTL mapping experiment. Moderate to relatively high heritability estimates were observed for different root traits (except RAD), indicating a better chance of reliably detecting QTL with large effects (Crespi, 2012).

GBS-SNP LINKAGE MAP. Because of their abundance and biallelic nature, SNP markers are popular for mapping studies. The GBS method applied in this tomato interspecific population yielded $\approx 1000$ markers, which was sufficient to generate a fairly dense genetic map. The SNP markers were not evenly distributed across the genome, with more on chromosome distal ends and few on the pericentromeric regions, showing similar features in other species genomic studies (Chen et al., 2014). Also, we used a methylation-sensitive enzyme, NgoMIV, for constructing the GBS libraries to avoid repetitive regions, which often locate in the gene-poor pericentromeric heterochromatin. It may therefore exclude potential markers in those regions. In the present study, segregation distortion was observed on chromosomes 4 and 11, with a decreased frequency of Lche4 alleles, which was also found in previous mapping studies of $S$. lycopersicum and $S$. cheesmaniae (Paterson et al., 1991). This segregation distortion presumably happened because of the selective abortion of gametes containing Lche4 alleles, suggesting the genomic regions on chromosomes 4 and 11 might have some unfavorable genetic factors. It may also suggest pollen that carries the Lche4 alleles could be viable but less competitive than pollen that carries Rvt1 alleles. Through careful screening procedures, the overall genotyping error rate for the chosen 742 SNP markers was reduced to $2.50 \%$ by maximum likelihood estimation (Supplemental Fig. 3). This percentage would not be an issue in performing downstream QTL analysis. A genetic map of the interspecific $\mathrm{F}_{2}$ population from $S$. lycopersicum and $S$. cheesmaniae was initially developed by Paterson et al. (1991) using 71 RFLP markers, spanning $1023 \mathrm{cM}$. Compared with this linkage map, our GBS-SNP map reduced the distance between adjacent markers from $17.3 \mathrm{cM}$ to $1.78 \mathrm{cM}$. A more recent and comparable linkage map was constructed between S. lycopersicum and Solanum galapagense by Firdaus et al. (2013), using 589 SNP markers in a population of $181 \mathrm{~F}_{2}$ seedlings, which spanned $1259 \mathrm{cM}$ with a resolution of $2.13 \mathrm{cM}$ per marker. Compared with this map, our map was slightly shorter but more precise in resolution because more markers were included. Generally, our map was medium dense compared with other tomato linkage maps such as EXPEN2000 and EXPEN2012 that consist of 3503 and 3687 markers, respectively (Sim et al., 2012).

QTLS IDENTIFIED FOR ROOT TRAITS. In this study, 15 QTLs were identified for root traits in the tomato $\mathrm{F}_{2}$ population RvT1x Lche4. Among them, six were major QTLs, and nine were minor QTLs. Considering the low to moderate phenotypic variance explained by each QTL, improvement in root traits might be achieved by accumulating multiple positive alleles at different loci. Detected root QTLs in this interspecific population were mainly partially or totally dominant but rarely additive (Table 4). The alleles of wild species Lche4 were inferior to the alleles of cultivated RvT1 for root mass traits but 


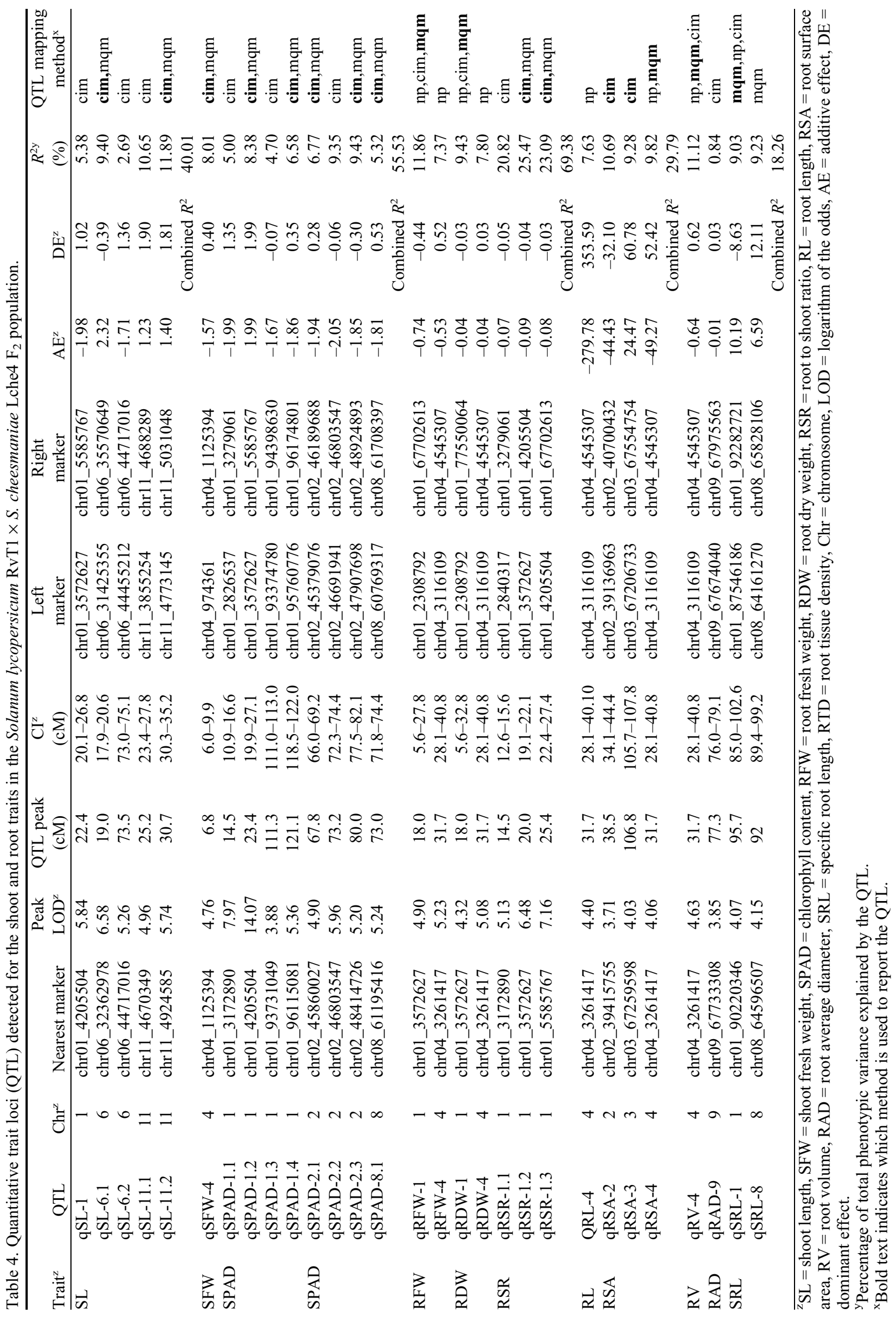


superior for SRL. Markers flanking QTL of SRL, including additive qSRL-1 and partially dominant qSRL-8, could be used to integrate these genomic regions of Lche4 into RvT1 to modify the root system in a positive way in breeding programs. The QTL cluster, which includes five root QTLs, was closely linked with QTLs of SL and SPAD on chromosome 1 (Fig. 6). This genetic relationship confirmed that these three root mass traits (RDW, RFW, and RSR) were moderately positively correlated with SL and SPAD (Fig. 2). The second QTL cluster on chromosome 4 was consistent with a QTL hotspot region associated with root length, root area, and stele area, which was identified using introgression lines 4-2 derived from $S$. lycopersicum and S. pennelli (Ron et al., 2013).

Two QTLs of SRL contributed by Lche4 were tightly linked with SPAD QTLs contributed by RvT1. There will be a compromise when selecting them simultaneously because it is hard to break such tight linkage by recombination. However, QTL for root and shoot traits were not always on the same linkage group, providing a possibility for independent selection. One QTL of SRL on chromosome 8 was separated from two QTL clusters of root mass traits on chromosomes 1 and 4, providing an opportunity to select an improved root system by combining a large root biomass with a high specific root length.

In this study, two QTL hotspot regions for root traits were identified on chromosomes 1 and 4. Those two regions share common markers and could be useful for screening larger roots and shoots. Our results indicate the possibility of selecting genotypes that combine acceptable shoot mass with improved root systems (higher SRL), which provides new insight for plant breeders who are targeting root traits, although using an $\mathrm{F}_{2}$ population that cannot be replicated is a drawback in terms of stability of QTL. To develop stable markers for root and shoot traits in this population, we are currently developing RILs from each $F_{2}$ individual seedling. QTLs and associated markers in this study will be validated for multiple environments and years by using RILs.

\section{Literature Cited}

Ali, M.L., M.S. Pathan, J. Zhang, G. Bai, S. Sarkarung, and H.T. Nguyen. 2000. Mapping QTLs for root traits in a recombinant inbred population from two indica ecotypes in rice. Theor. Appl. Genet. 101:756-766.

Bai, C., Y. Liang, and M.J. Hawkesford. 2013. Identification of QTLs associated with seedling root traits and their correlation with plant height in wheat. J. Expt. Bot. 64:1745-1753.

Broman, K.W., H. Wu, S. Sen, and G.A. Churchill. 2003. R/qtl: QTL mapping in experimental crosses. Bioinformatics 19:889-890.

Broman, K.W. and S. Sen. 2009. A guide to QTL mapping with R/qtl. Vol. 46. Springer, New York, NY.

Burton, A.L., J.M. Johnson, J.M. Foerster, C.N. Hirsch, C.R. Buell, M.T. Hanlon, S.M. Kaeppler, K.M. Brown, and J.P. Lynch. 2014. QTL mapping and phenotypic variation for root architectural traits in maize (Zea mays L.). Theor. Appl. Genet. 127:2293-2311.

Cai, H., F. Chen, G. Mi, F. Zhang, H.P. Maurer, W. Liu, J.C. Reif, and L. Yuan. 2012. Mapping QTLs for root system architecture of maize (Zea mays L.) in the field at different developmental stages. Theor. Appl. Genet. 125:1313-1324.

Champoux, M.C., G. Wang, S. Sarkarung, D.J. Mackill, J.C. O'Toole, N. Huang, and S.R. McCouch. 1995. Locating genes associated with root morphology and drought avoidance in rice via linkage to molecular markers. Theor. Appl. Genet. 90:969-981.

Chen, A.L., C.Y. Liu, C.H. Chen, J.F. Wang, Y.C. Liao, C.H. Chang, M.H. Tsai, K.K. Hwu, and K.Y. Chen. 2014. Reassessment of QTLs for late blight resistance in the tomato accession L3708 using a restriction site associated DNA (RAD) linkage map and highly aggressive isolates of Phytophthora infestans. PLoS One 9:e96417.

Churchill, G.A. and R.W. Doerge. 1994. Empirical threshold values for quantitative trait mapping. Genetics 138:963-971.

Coudert, Y., C. Périn, B. Courtois, N.G. Khong, and P. Gantet. 2010. Genetic control of root development in rice, the model cereal. Trends Plant Sci. 15:219-226.

Crespi, M. (ed.). 2012. Root genomics and soil interactions. Wiley, New York, NY.

Eissenstat, D.M. 1991. On the relationship between specific root length and the rate of root proliferation: A field study using citrus rootstocks. New Phytol. 118:63-68.

Elshire, R.J., J.C. Glaubitz, Q. Sun, J.A. Poland, K. Kawamoto, E.S. Buckler, and S.E. Mitchell. 2011. A robust, simple genotyping-bysequencing (GBS) approach for high diversity species. PLoS One 6:e19379.

Firdaus, S., A.W. van Heusden, N. Hidayati, E.D.J. Supena, R. Mumm, R.C. de Vos, R.G. Visser, and B. Vosman. 2013. Identification and QTL mapping of whitefly resistance components in Solanum galapagense. Theor. Appl. Genet. 126:1487-1501.

Foolad, M.R. 2007. Genome mapping and molecular breeding of tomato. Int. J. Plant Genomics 2007:64358.

Food and Agriculture Organization of the United Nations. 2017. FAOSTAT crops data. 8 Mar. 2019. <http://www.fao.org/faostat/ en/?\#data/QC>.

Fulton, T.M., J. Chunwongse, and S.D. Tanksley. 1995. Microprep protocol for extraction of DNA from tomato and other herbaceous plants. Plant Mol. Biol. Rpt. 13:207-209.

Goldman, I., I. Paran, and D. Zamir. 1995. Quantitative trait locus analysis of a recombinant inbred line population derived from a Lycopersicon esculentum $\times$ Lycopersicon cheesmanii cross. Theor. Appl. Genet. 90:925-932.

Hajek, P., D. Hertel, and C. Leuschner. 2013. Intraspecific variation in root and leaf traits and leaf-root trait linkages in eight aspen demes (Populus tremula and P. tremuloides). Front. Plant Sci. 4:415.

Kosambi, D.D. 1943. The estimation of map distances from recombination values. Ann. Eugen. 12:172-175.

Kuijken, R.C., F.A. van Eeuwijk, L.F. Marcelis, and H.J. Bouwmeester. 2015. Root phenotyping: From component trait in the lab to breeding. J. Expt. Bot. 66:5389-5401.

Liu, J., J. Li, F. Chen, F. Zhang, T. Ren, Z. Zhuang, and G. Mi. 2008. Mapping QTLs for root traits under different nitrate levels at the seedling stage in maize (Zea mays L.). Plant Soil 305:253-265.

Liu, X., R. Li, X. Chang, and R. Jing. 2013. Mapping QTLs for seedling root traits in a doubled haploid wheat population under different water regimes. Euphytica 189:51-66.

Manavalan, L.P., S.J. Prince, T.A. Musket, J. Chaky, R. Deshmukh, T.D. Vuong, L. Song, P.B. Cregan, J.C. Nelson, J.G. Shannon, and J.E. Specht. 2015. Identification of novel QTL governing root architectural traits in an interspecific soybean population. PLoS One 10:e0120490.

Monje, O.A. and B. Bugbee. 1992. Inherent limitations of nondestructive chlorophyll meters: A comparison of two types of meters. HortScience 27:69-71.

Morishige, D.T., P.E. Klein, J.L. Hilley, S.M.E. Sahraeian, A. Sharma, and J.E. Mullet. 2013. Digital genotyping of sorghum-A diverse plant species with a large repeat-rich genome. BMC Genomics $14: 448$.

Nuez, F., J. Prohens, and J.M. Blanca. 2004. Relationships, origin, and diversity of Galapagos tomatoes: Implications for the conservation of natural populations. Amer. J. Bot. 91:86-99.

Paterson, A.H., S. Damon, J.D. Hewitt, D. Zamir, H.D. Rabinowitch, S.E. Lincoln, E.S. Lander, and S.D. Tanksley. 1991. Mendelian factors underlying quantitative traits in tomato: Comparison across species, generations, and environments. Genetics 127:181-197.

Rakha, M., P. Hanson, and S. Ramasamy. 2017. Identification of resistance to Bemisia tabaci Genn. in closely related wild relatives of 
cultivated tomato based on trichome type analysis and choice and nochoice assays. Genet. Resources Crop Evol. 64:247-260.

Ren, Y., X. He, D. Liu, J. Li, X. Zhao, B. Li, Y. Tong, A. Zhang, and Z. Li. 2012. Major quantitative trait loci for seminal root morphology of wheat seedlings. Mol. Breed. 30:139-148.

Ron, M., M.W. Dorrity, M. de Lucas, T. Toal, R.I. Hernandez, S.A. Little, J.N. Maloof, D.J. Kliebenstein, and S.M. Brady. 2013. Identification of novel loci regulating interspecific variation in root morphology and cellular development in tomato. Plant Physiol. 162:755-768.

Ruta, N., M. Liedgens, Y. Fracheboud, P. Stamp, and A. Hund. 2010. QTLs for the elongation of axile and lateral roots of maize in response to low water potential. Theor. Appl. Genet. 120:621-631. Ryser, P. 2006. The mysterious root length. Plant Soil 286:1-6.

Sim, S.C., G. Durstewitz, J. Plieske, R. Wieseke, M.W. Ganal, A. Van Deynze, J.P. Hamilton, C.R. Buell, M. Causse, S. Wijeratne, and D.M. Francis. 2012. Development of a large SNP genotyping array and generation of high-density genetic maps in tomato. PLoS One 7:e40563.

Spindel, J., M. Wright, C. Chen, J. Cobb, J. Gage, S. Harrington, M. Lorieux, N. Ahmadi, and S. McCouch. 2013. Bridging the genotyping gap: Using genotyping by sequencing (GBS) to add high-density SNP markers and new value to traditional bi-parental mapping and breeding populations. Theor. Appl. Genet. 126:2699-2716.

Tang, Y., M. Horikoshi, and W. Li. 2016. ggfortify: Unified interface to visualize statistical results of popular R packages. R J. 8:478-489.

Tanksley, S.D., M.W. Ganal, J.P. Prince, M.C. De Vicente, M.W. Bonierbale, P. Broun, T.M. Fulton, J.J. Giovannoni, S. Grandillo, and G.B. Martin. 1992. High density molecular linkage maps of the tomato and potato genomes. Genetics 132:1141-1160.

Taylor, J. and D. Butler. 2014. ASMap: Linkage map construction using the MSTmap algorithm. R package version 0.4-5. 15 Mar. 2017. <https://cran.r-project.org/web/packages/ASMap/>.
Tomato Genome Consortium. 2012. The tomato genome sequence provides insights into fleshy fruit evolution. Nature 485:635-641.

Topp, C.N., A.S. Iyer-Pascuzzi, J.T. Anderson, C.R. Lee, P.R. Zurek, O. Symonova, Y. Zheng, A. Bucksch, Y. Mileyko, T. Galkovskyi, and B.T. Moore. 2013. 3D phenotyping and quantitative trait locus mapping identify core regions of the rice genome controlling root architecture. Proc. Natl. Acad. Sci. USA 110:1695-1704.

Truco, M.J., L.B. Randall, A.J. Bloom, and D.A. St. Clair. 2000. Detection of QTLs associated with shoot wilting and root ammonium uptake under chilling temperatures in an interspecific backcross population from Lycopersicon esculentum $\times L$. hirsutum. Theor. Appl. Genet. 101:1082-1092.

Uga, Y., K. Okuno, and M. Yano. 2011. Dro1, a major QTL involved in deep rooting of rice under upland field conditions. J. Expt. Bot. 62:2485-2494.

Uga, Y., K. Sugimoto, S. Ogawa, J. Rane, M. Ishitani, N. Hara, Y. Kitomi, Y. Inukai, K. Ono, N. Kanno, and H. Inoue. 2013. Control of root system architecture by DEEPER ROOTING 1 increases rice yield under drought conditions. Nat. Genet. 45:1097-1102.

Van Ooijen, J.W. 2006. JoinMap 4.0: Software for the calculation of genetic linkage maps in experimental populations. Kyazma, Wageningen, Netherlands.

Wang, S., C.J. Basten, and Z.B. Zeng. 2011. Windows QTL Cartographer 2.5. Dept. Statistics, North Carolina State Univ., Raleigh.

Wei, T. and V. Simko. 2013. corrplot: Visualization of a correlation matrix. $\mathrm{R}$ package version 0.73. 15 Mar. 2017. <https://cran. rproject.org/web/packages/corrplot/index.html>.

Weng, Y., M. Colle, Y. Wang, L. Yang, M. Rubinstein, A. Sherman, R. Ophir, and R. Grumet. 2015. QTL mapping in multiple populations and development stages reveals dynamic quantitative trait loci for fruit size in cucumbers of different market classes. Theor. Appl. Genet. 128:1747-1763. 


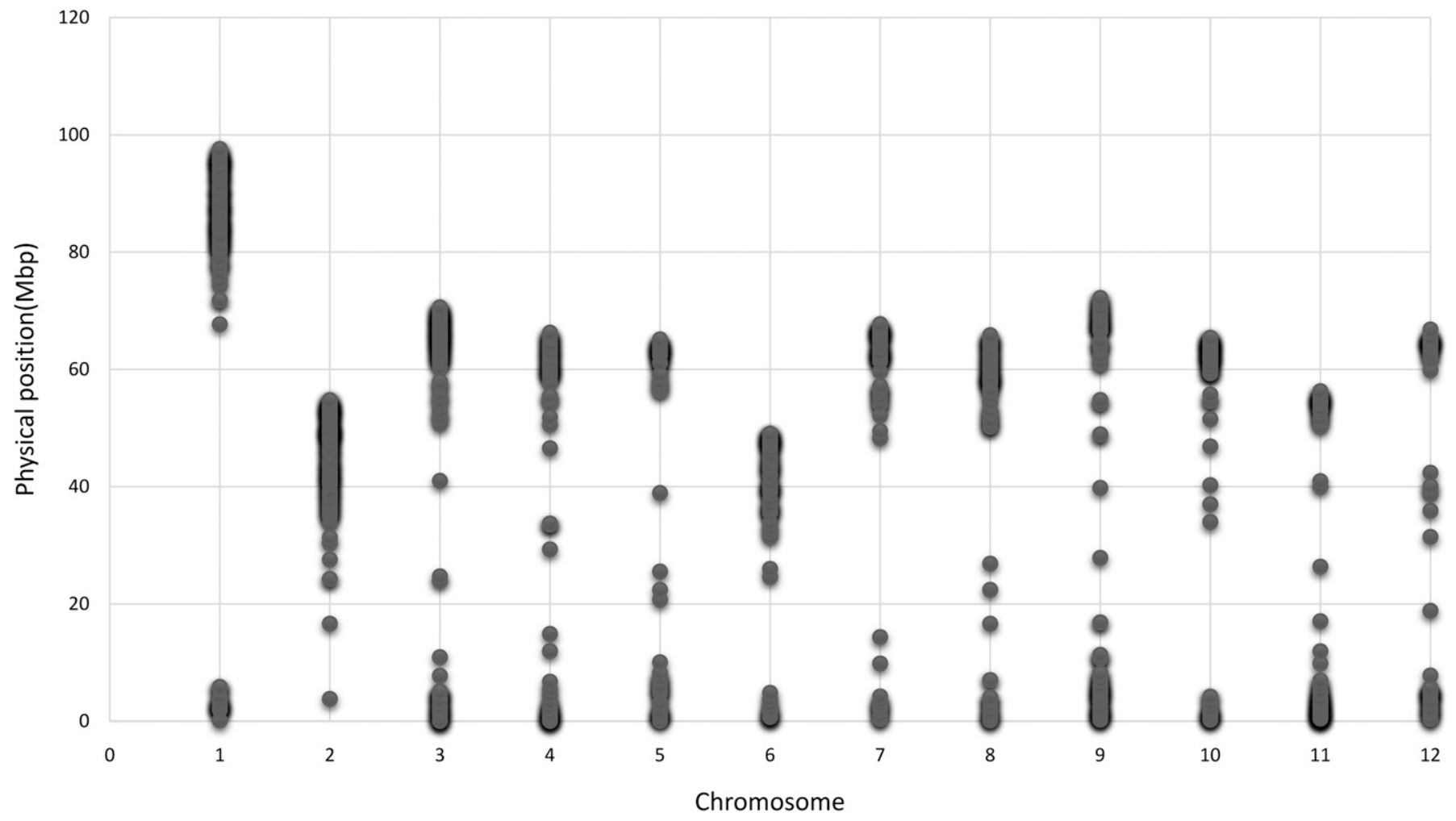

Supplemental Fig. 1. Physical map of 1002 single-nucleotide-polymorphism markers distributed on 12 chromosomes in the Solanum lycopersicum RvT1 $\times$ S. cheesmaniae Lche4 $\mathrm{F}_{2}$ population $(\mathrm{n}=181)$. 


\section{Chr1 Chr2 Chr3 Chr4 Chr5 Chr6 Chr7 Chr8 Chr9 Chr10 Chr11 Chr12}
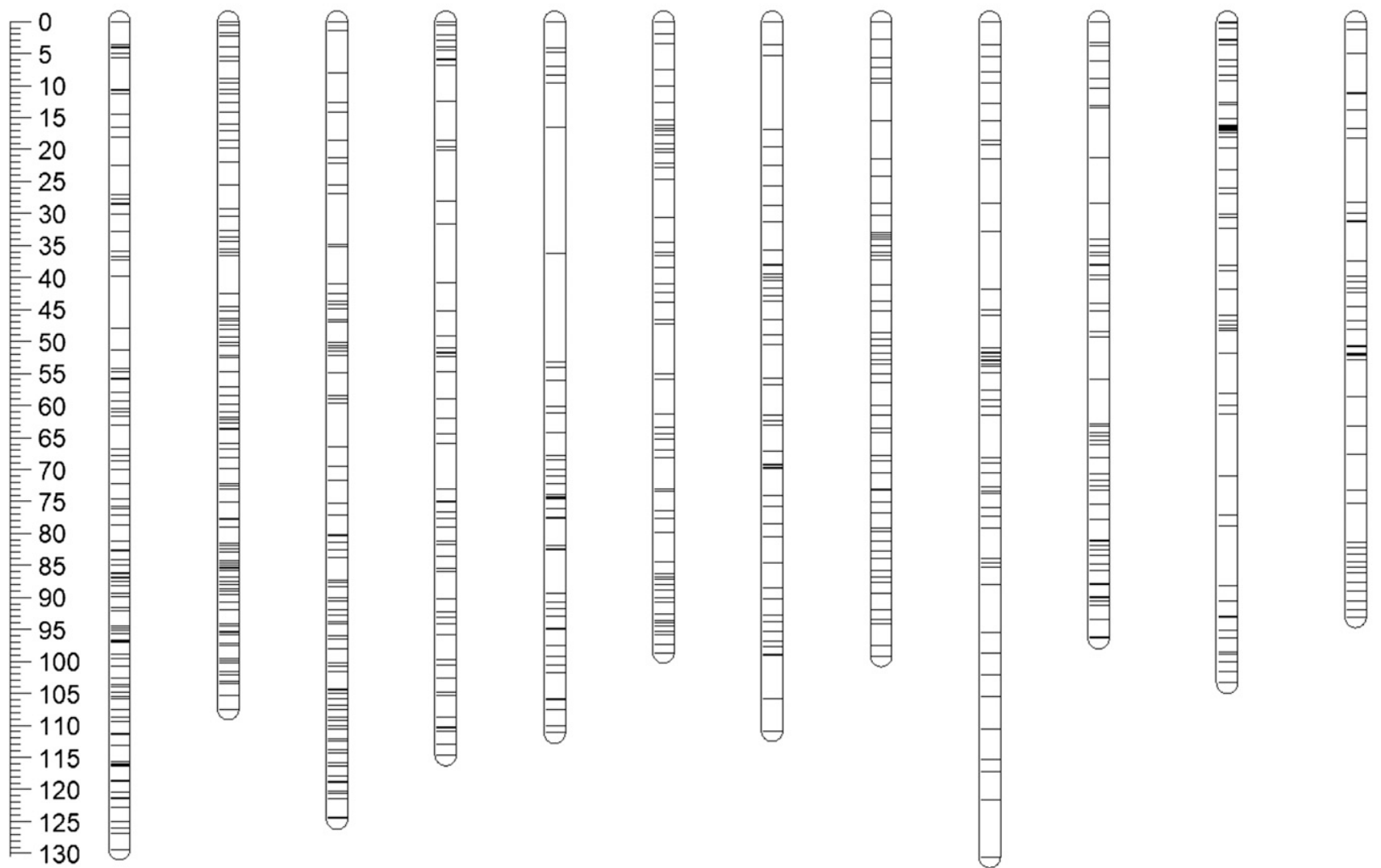

Supplemental Fig. 2. Genetic map of the Solanum lycopersicum RvT1 $\times$ S. cheesmaniae Lche4 $\mathrm{F}_{2}$ population $(\mathrm{n}=181)$; 742 single-nucleotide-polymorphism markers mapped on 12 chromosomes (Chr), which spanned $1319.47 \mathrm{cM}$ with an average genetic interval of $1.78 \mathrm{cM}$. 


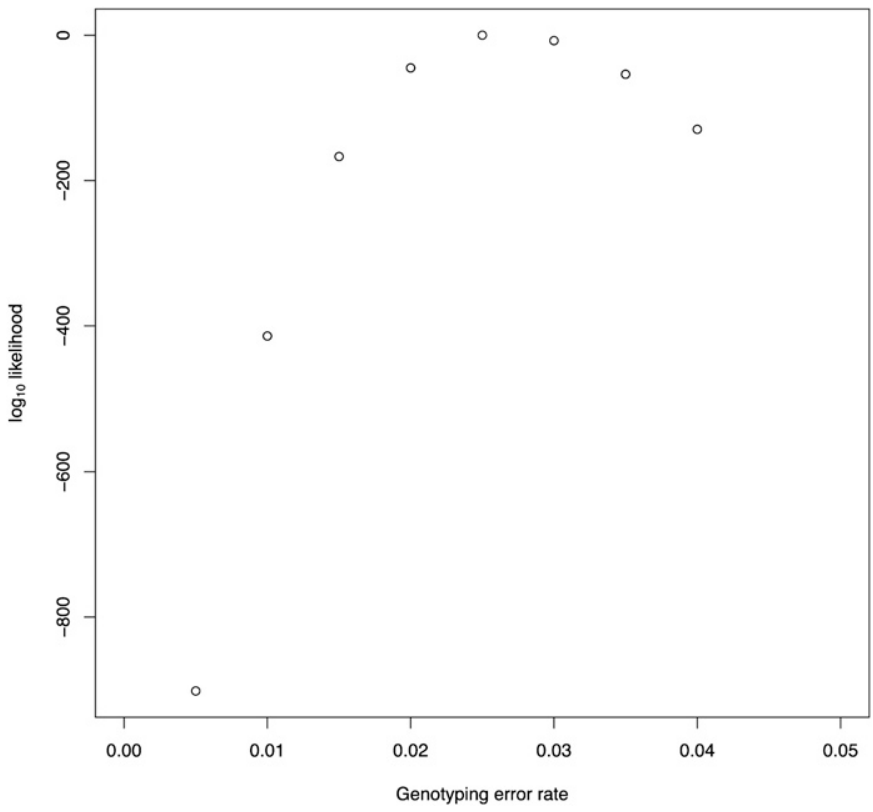

Supplemental Fig. 3. Genotyping error rate of the Solanum lycopersicum RvT1 $\times$ S. cheesmaniae Lche4 $\mathrm{F}_{2}$ population $(\mathrm{n}=181)$ estimated by maximum likelihood method.

Supplemental Table 1. Mean value of shoot and root traits of 9-week-old seedlings of Solanum lycopersicum RvT1 ( $\mathrm{n}=13)$ and S. cheesmaniae Lche4 $(\mathrm{n}=9)$.

\begin{tabular}{|c|c|c|c|c|c|c|}
\hline & Shoot length $(\mathrm{cm})$ & Shoot fresh wt $(\mathrm{g})$ & Root length $(\mathrm{cm})$ & Root surface area $\left(\mathrm{cm}^{2}\right)$ & Root vol $\left(\mathrm{cm}^{3}\right)$ & Root avg diam (mm) \\
\hline RvT1 & 23.5 & 16.4 & 603.26 & 153.39 & 3.13 & 0.87 \\
\hline Lche4 & 29.28 & 7.55 & 344.1 & 79 & 1.46 & 0.71 \\
\hline $\begin{array}{c}\text { Significant } \\
\text { level }\end{array}$ & NS & $*$ & $*$ & $*$ & $*$ & $*$ \\
\hline
\end{tabular}

Supplemental Table 2. Shapiro-Wilk Test of shoot and root traits frequency distribution in the Solanum lycopersicum $\mathrm{RvT} 1 \times S$. cheesmaniae Lche4 $\mathrm{F}_{2}$ population $(\mathrm{n}=181)$.

\begin{tabular}{lc}
\hline Phenotype & $P$ \\
\hline Shoot length & 0.22 \\
Shoot fresh wt & 0.29 \\
SPAD & 0.89 \\
Root fresh wt & $<0.001$ \\
Root dry wt & $<0.001$ \\
Root-to-shoot ratio & $<0.001$ \\
Root length & $<0.001$ \\
Root surface area & $<0.001$ \\
Root average diameter & 0.69 \\
Root volume & $<0.001$ \\
Specific root length & 0.02 \\
Root tissue density & $<0.001$ \\
\hline
\end{tabular}

